\title{
Assessment of Quadratic Nonlinear Cardiorespiratory Couplings During Tilt Table Test \\ by Means of Real Wavelet Biphase
}

\author{
Spyridon Kontaxis ${ }^{1}$, Jesús Lázaro ${ }^{2}$, Eduardo Gil ${ }^{1}$, Pablo Laguna ${ }^{1}$, Raquel Bailón ${ }^{1}$ \\ ${ }^{1}$ Biomedical Signal Interpretation and Computational Simulation (BSICoS) \\ Institute of Engineering Research (I3A), IIS Aragón, University of Zaragoza, \\ and CIBER de Bioingeniería, Biomateriales y Nanomedicina (CIBER-BBN),Spain. \\ e-mail: sikontax@gmail.com \\ ${ }^{2}$ Department of Electrical Engineering (ESAT), STADIUS \\ Centre for Dynamical Systems, Signal Processing and Data Analytics, KU \\ Leuven, Leuven, Belgium and IMEC, Leuven, Belgium.
}

\begin{abstract}
In this paper a method for the assessment of Quadratic Phase Coupling (QPC) between respiration and Heart Rate Variability is presented and applied to study cardiorespiratory couplings during a tilt table test. Strong QPC related to the dominant respiratory frequency is present and remains unchanged during Autonomic Nervous System changes.
\end{abstract}

\section{Introduction}

The coupling of heart rate (HR) to the respiratory cycle has been extensively studied as the heart period decreases during inspiration and increases during expiration [1]. Heart rate variability (HRV) has been proposed as a noninvasive indicator of autonomic nervous system (ANS) regulation over the heart and it is often considered to have a complex structure involving various superimposed oscillation frequencies, non-linearly related to each other. A variety of studies have been implemented to analyze the nonlinear properties of HRV signals and to reveal time-phase relationships among cardiorespiratory oscillations with quadratic nature [2].

\section{Methods for QPC detection}

A simple non-linearity introduces new harmonics with higher-order frequency and phase correlations which simply could not have been introduced through a linear transformation. The normalized bispectrum, known as wavelet bicoherence (WB) is proposed to quantify not linearities and is defined as [3]:
$b_{W}^{2}\left(f_{1}, f_{2}\right)=\frac{\left(\int_{T} W_{x}\left(f_{1}, \tau\right) W_{x}\left(f_{2}, \tau\right) W_{x}^{*}\left(f_{12}, \tau\right) d \tau\right)^{2}}{\int_{T}\left|W_{x}\left(f_{1}, \tau\right) W_{x}\left(f_{2}, \tau\right)\right|^{2} d \tau \int_{T}\left|W_{x}\left(f_{12}, \tau\right)\right|^{2} d \tau}$

where $f_{12}=f_{1}+f_{2}$ and $W_{x}(f, t)$ is the continuous wavelet transform for the input signal $x(t)$. The numerator is know as bispectrum. A direct application of bicoherence magnitude as a specific measure of QPC may cause incorrect results when biphase relationship is constant across the integration time (Quadratic Phase Uncoupling QPU) but not zero which ensures QPC. Biphase randomization wavelet bicoherence (BRWB) [4], multiplying bispectrum with a biphase randomization term for multiple realizations and (ensemble average) reduces bicoherence when the biphase is not close to 0 but constant across the segments (QPU). Considering that the wavelet biphase carries the information about the nature of quadratic coupling, we propose a new detector named Real Waletet Biphase (RWB) based on the instantaneous wavelet biphase. The values close to 1 correspond to the perfect QPC while other values correspond to the deflection from it.

\section{Cardiorespiratory RWB}

RWB is used to study nonlinear cardiorespiratory interactions during ANS response to a tilt test (seventeen volunteers, 4 minutes in early supine position, 5 minutes headup tilted and 4 minutes back to later supine position). Our hypothesis is that HRV signal might be formed as an output of a non-linear system where a low frequency oscillation could represent a component of sympathetic and parasympathetic activation and another oscillation related to the respiration that controls HRV not only through a linear function but also with a non-linear

Revista "Jornada de Jóvenes Investigadores del I3A", vol. 5 (Actas de la VI Jornada de Jóvenes Investigadores del I3A - 2 de junio de 2017). ISSN 2341-4790. 
one. Note that previous methods were defined to analyze onesignal but can be extended to study nonlinear cross-phase relationships between different signals.

When the dominant respiratory oscillation appear in $\mathrm{HRV}$ as a result of a quadratic relationship, a representative feature is the maximum value of the points located in the diagonal line $\left(f_{1}=f_{2}\right)$ or in a narrow range around of it, as well as the time percentage in which the estimated coupling was higher than 0.75 which corresponds to a strong nonlinear coupling in the analyzed interval.

\section{Simulation study}

A simulation was carried out to evaluate the performance of WB, BRWB and RWB. It offers the ability to investigate the correct detection of QPC, and its reliability in cases of linear and QPU cases.

$$
\begin{gathered}
y_{R}(t)=\cos \left(2 \pi f_{r} t+\varphi_{r}\right)+n_{y}(t) \\
x_{H R V}(t)=y_{R}(t)+\frac{\varepsilon}{2} \cos \left(2 \pi 2 f_{r} t+\varphi_{x}\right)+n_{x}(t)
\end{gathered}
$$

where $f_{r}=0.25 \mathrm{~Hz}$ and $\varphi_{r} \in[-\pi, \pi]$. Linear cardiorespiratory relationships are simulated with $\varepsilon=0$, and the nonlinear ones with $\varepsilon=1$ and $\varphi_{x}=2 \varphi_{r}$ (QPC) which ensures zero biphase. In case of constant phase relationships (QPU), a non-zero biphase is simulated with $\varepsilon=1$ and $\varphi_{x} \in[-\pi, \pi]$.

\section{Results}

Results from simulation showed that WB fails to discriminate between QPC waves and constant phase relationships (QPU) since high bicoherence levels are present for both of them. BRWB and RWB get high value in QPC and lower values in linear relationships and QPU.

Results from the tilt table database suggest that strong QPC related to the dominant respiratory frequency $\left(f_{r}\right)$ is present for a high time percentage and remains unchanged during ANS changes induced by tilt table maneuver for all the subjects.

\section{REFERENCES}

[1]. YAMSUMA F, HAYANO JI. Respiratory sinus arrhythmia: why does the heartbeat synchronize with respiratory rhythm?. Chest Journal. 2004 Feb 1;125(2):683-90.

[2]. JAMSEK J, STEFANOVSKA A, MCCLINTOCK $\mathrm{PV}$. Nonlinear cardio-respiratory interactions revealed by time-phase bispectral analysis. Physics in medicine and biology. 2004 Sep 3;49(18):4407.

[3]. VAN MILLIGEN BP, SANCHEZ E, ESTRADA T, HIDALGO C, BRAÑAS B, CARRERAS B, GARCIA L. Wavelet bicoherence: a new turbulence analysis tool. Physics of Plasmas. 1995 Aug;2(8):3017-32.

[4]. LI Y, LIN J, WANG X, LEI Y. Biphase randomization wavelet bicoherence for mechanical fault diagnosis. Measurement. 2014 Mar 31;49:40720. 